\title{
Environmental Monitoring During Decommissioning of a CNT Manufacturing Facility Using Polarized Light Microscopy and FE-SEM
}

\author{
E.F. Schumacher,* S.F. Stoeffler,* C.S. Schwandt,* and K.J. Diebold* \\ * McCrone Associates, Inc., 850 Pasquinelli Drive, Westmont, IL 60559-5539
}

Commercial scale manufacturing of carbon nanotubes (CNTs) and their incorporation into a variety of materials are rapidly on the rise. Concerns about industrial hygiene, consumer safety and environmental impact accompany the proliferation of CNTs and other nanoscale materials in commonly used products and in construction materials. Methods for confirming, and ideally, quantifying presence of CNTs in the workplace and the larger environment are needed so that exposure can be measured and regulations put in place to protect workers and consumers who come into contact with these materials [1].

Due to their small size, CNTs are typically identified using a high resolution characterization technique such as transmission electron microscopy (TEM) or field emission scanning electron microscopy (FE-SEM). However, because of the time required to representatively analyze samples at the high magnifications required for CNT identification, such techniques are not well suited for rapid screening of large amounts of material, especially for samples consisting of mixtures of particulate typically found in industrial and environmental collections. Presence of background particulate can also preclude the use of fingerprint or bulk techniques such as Raman spectroscopy to identify CNTs, or elemental analysis for identification of CNT metal catalyst particles in a mixed particulate sample such as a wipe collected from a surface in an industrial facility.

Four studies were carried out for an industrial hygiene consulting company whose client, a commercial CNT producer, was decommissioning a CNT manufacturing facility. Before the decommissioning process began, a baseline study was done to qualitatively map presence of CNTs throughout the building. Subsequent studies monitored cleanliness and worker exposure during removal of the CNT manufacturing equipment and final cleaning of the facility. Fast turnaround was required to ensure that the contract workers doing the cleaning would not be idle while waiting for results to determine whether a second round of cleaning was required for each room tested. Scheduling for mold remediation being done concurrently during the final cleanup also depended upon provision of result within a few days.

Using a reference sample of the producer's CNT material, a methodology was developed whereby wipes or air filters were first examined using polarized light microscopy (PLM). The substrates were assessed for presence or absence of black particulate, and when present, the black particulate was qualitatively rated for its similarity in appearance to the reference CNT sample, as shown in Fig. 1. Substrates supporting particulate with little or no similarity to the reference material did not undergo any further testing. Where any similarity to the reference was noted, the suspect black particulate was directly isolated in a cleanroom and transferred to a beryllium substrate for examination using FE-SEM. In a few cases, TEM was also used to verify presence of CNTs. Targeting only the suspect black particulate for isolation greatly reduced the amount of material that had to be examined using high resolution techniques. The methodology, outlined in Fig. 2, is 
analogous to use of PLM for preliminary identification of the aciniform, or grape-cluster morphology characteristic of soot, followed by TEM analysis to complete the identification process.

Though not typically considered as a method for identification of nanomaterials, PLM proved valuable for rapid screening for presence of CNTs in a large number of samples consisting of mixed particulate. In the course of the four studies, 83 wipes and 30 air filters were examined, and PLM indicated possible presence of CNTs in 60 out of 113 samples. Using FE-SEM and TEM, presence of CNTs was confirmed in 34 out of the 60 samples. Repeat sampling and SEM analysis of several wipes gave consistent results, heightening confidence in the screening methodology [2].

\section{References}

[1] A.D. Maynard et al., Journal of Nanoparticle Research. 9 (2007) 85-92.

[2] We would like to thank ICU Environmental, Health and Safety Consultants and Unidym, Inc. for granting their permission to present these results.

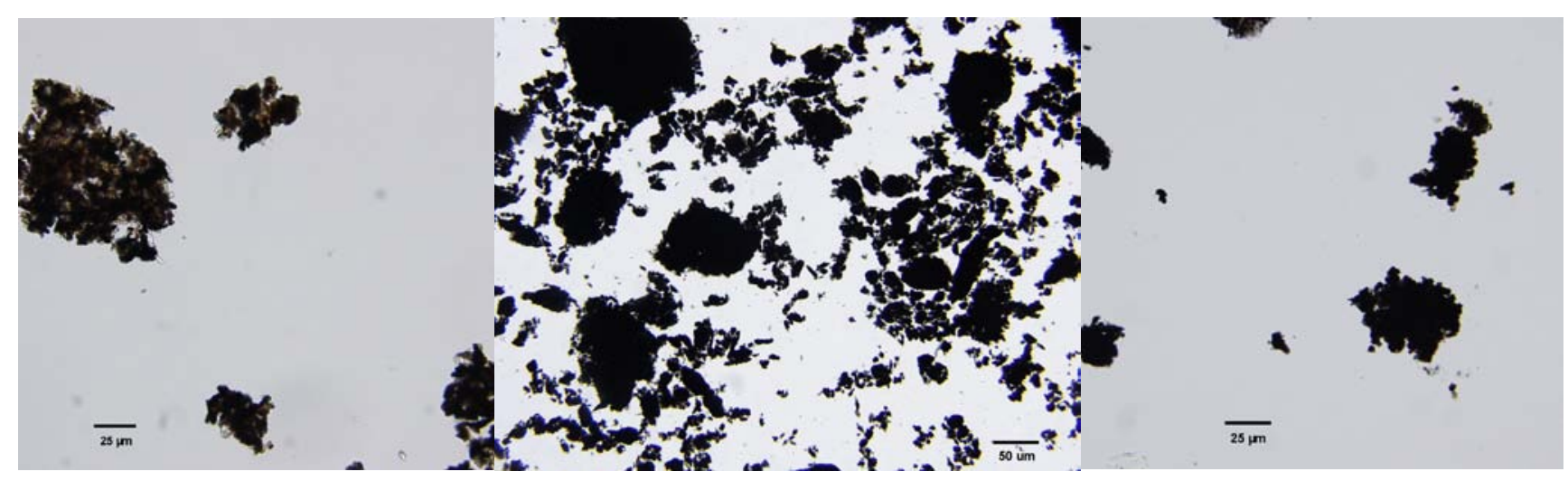

FIG. 1. PLM photomicrographs of reference CNT material (center) and wipe particulate with low similarity to reference (left) and possible similarity to reference (right). FE-SEM confirmed presence of CNTs in both samples of wipe particulate.
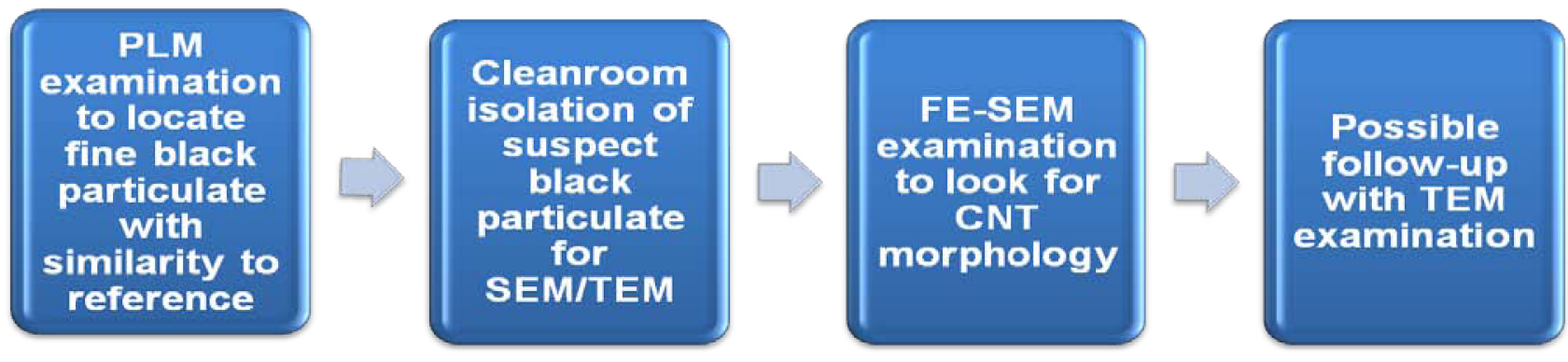

FIG. 2. Methodology for rapid screening of wipes and air filters suspected to support CNTs among mixed particulate. 